\title{
Task Deactivation Reductions and Atrophy within Parietal Default Mode Regions are Overlapping but Only Weakly Correlated in Mild Cognitive Impairment
}

\author{
Zachary D. Threlkeld ${ }^{\mathrm{a}}$, Greg A. Jicha ${ }^{\mathrm{b}, \mathrm{c}}$, Charles D. Smith ${ }^{\mathrm{a}, \mathrm{b}, \mathrm{c}, \mathrm{d}}$ and Brian T. Gold ${ }^{\mathrm{a}, \mathrm{c}, \mathrm{d}, *}$ \\ ${ }^{a}$ Departments of Anatomy and Neurobiology, University of Kentucky, Lexington, KY, USA \\ ${ }^{\mathrm{b}}$ Departments of Neurology, University of Kentucky, Lexington, KY, USA \\ 'Sanders-Brown Center on Aging and Alzheimer's Disease Center, University of Kentucky, Lexington, KY, USA \\ ${ }^{\mathrm{d}}$ Magnetic Resonance Imaging and Spectroscopy Center, Chandler Medical Center, University of Kentucky, \\ Lexington, $K Y$, USA
}

Handling Associate Editor: Christine Bastin

Accepted 22 June 2011

\begin{abstract}
Reduced task deactivation within regions of the default mode network (DMN) has been frequently reported in Alzheimer's disease (AD) and amnestic mild cognitive impairment (aMCI). As task deactivation reductions become increasingly used in the study of early AD states, it is important to understand their relationship to atrophy. To address this issue, the present study compared task deactivation reductions during a lexical decision task and atrophy in aMCI, using a series of parallel voxel-wise and region-wise analyses of fMRI and structural data. Our results identified multiple regions within parietal cortex as convergence areas of task deactivation and atrophy in aMCI. Relationships between parietal regions showing overlapping task deactivation reductions and atrophy in aMCI were then explored. Regression analyses demonstrated minimal correlation between task deactivation reductions and either local or global atrophy in aMCI. In addition, a logistic regression model which combined task deactivation reductions and atrophy in parietal DMN regions showed higher classificatory accuracy of aMCI than separate task deactivation or atrophy models. Results suggest that task deactivation reductions and atrophy in parietal regions provide complementary rather than redundant information in aMCI. Future longitudinal studies will be required to assess the utility of combining task deactivation reductions and atrophy in the detection of early AD.
\end{abstract}

Keywords: Alzheimer's disease, atrophy, deactivation, default mode network (DMN), mild cognitive impairment, voxel-based morphometry

\section{INTRODUCTION}

Task deactivations in functional brain imaging studies reflect signal decreases during complex cognitive tasks compared to simpler baseline tasks [1-3]. Such

${ }^{*}$ Correspondence to: Brian T. Gold, Department of Anatomy and Neurobiology, University of Kentucky School of Medicine, Lexington, KY, 40536-0298, USA. Tel.: +(859) 323 4813; Fax: +(859) 257 6700; E-mail: brian.gold@uky.edu. deactivations have been reported across a range of cognitive tasks and stimulus sets in large portions of parietal cortex, including precuneus and lateral parietal regions, as well as in medial prefrontal cortex [1-3]. These parietal and medial prefrontal regions showing prominent deactivation are thought to be part of a default-mode network (DMN), or a set of regions contributing to many forms of internally-directed thought including remembering the past, envisoning the future, considering the thoughts and perspectives of others 
$[4,5]$. Consistent with this view, similar parietal and prefrontal regions, as well as additional regions, show prominent activation during resting-states, which inherently involves internally directed attention [6-8].

Reduced task deactivation in relation to Alzheimer's disease (AD) was initially noted in cognitively normal seniors at high genetic risk for this disease [9]. A body of research has subsequently demonstrated decreased task deactivation in AD [10-12]. In addition, reduced task deactivation has been reported in amnestic mild cognitive impairment (aMCI), with the most robust alterations being observed in medial and lateral parietal cortices [11-13]. The reduction in aMCI may be particularly important in that aMCI has been shown to represent early-stage $\mathrm{AD}$ in the majority of cases that have come to autopsy or have been followed longitudinally to the point of transition to clinical dementia [14-16]. Approximately half of aMCI patients will convert to AD within 3-5 years [17]. Reduced deactivation in aMCI could thus be suggestive of early AD.

The failure to deactivate parietal DMN regions is such a robust and consistent finding in $\mathrm{AD}$ that it has potential to contribute to detection of $\mathrm{AD}$ and monitoring of its progression. The use of task deactivation reductions as a functional probe would be particularly relevant if reductions reflected metabolically-related dysfunction as opposed to gross tissue loss (in which case task deactivation reductions would be redundant with volumetric markers). Indirect support for a metabolic contribution to reduced task deactivation in $\mathrm{AD}$ comes from findings that DMN regions have previously been shown to exhibit prominent hypometabolism in $\mathrm{AD}[18,19]$ and even in normal seniors at high AD-risk [20], as measured by fluorodeoxyglucose positron emission tomography (FDG-PET). If task deactivation reductions reflected similar underlying metabolic or synaptic dysfunction, then fMRI-based probes would be a preferable tool to FDG-PET for disease detection and monitoring because the fMRI technique does not require exposure to radiation.

A largely separate body of data has reported that medial and lateral parietal DMN regions show prominent atrophy in AD [21-24] and aMCI [25-27]. However, few if any studies have explored quantitative relationships between DMN reductions (associated with altered task deactivation or resting-state activation) and atrophy. In two studies, local measures of atrophy were used as nuisance covariates in the analyses of resting-state activations [28, 29]. He and colleagues [28] reported that, after entering GM volumetric information as a nuisance covariate in fMRI analyses, DMN reductions in the precuneus of an $\mathrm{AD}$ group were reduced but remained significant. A similar approach was used by Bai et al. [29], who reported decreased DMN activation in the precuneus in aMCI despite the use of regional atrophy as a nuisance covariate (although fMRI results without atrophy as a covariate were not reported, preventing inference about the relationship between DMN activation and atrophy).

These resting-state studies are among the few to attempt to control for effects of local atrophy on local DMN reductions in neurodegenerative groups. However, these studies did not explore quantitative relationships between DMN reductions and atrophy. Similarly, no studies have explored quantitative relationships between task deactivations and atrophy in a common group of subjects. In the present study we compare fMRI deactivations associated with the performance of a lexical decision task and volumetric data in an aMCI group and normal senior group. The goals of the study were: 1) to identify potential locations of topographical overlap between task deactivations and atrophy in aMCI; 2) to compare the relative size of task deactivation reductions and volumetric reductions; 3 ) to assess potential relationships between task deactivations and local and global atrophy in aMCI; and 4) to determine the relative accuracy of task deactivation reductions and atrophy in classifying aMCI.

\section{MATERIALS AND METHODS}

\section{Participants}

The research procedures were approved by the Institutional Review Board of the University of Kentucky Medical Center, and all participants provided informed written consent. All participants were communitydwelling individuals who were right-handed, with normal or corrected-to-normal visual acuity, and were recruited from the University of Kentucky Alzheimer's Disease Center (UK-ADC) longitudinal normal volunteer cohort. Inclusion criteria for this cohort are minimum 65 years of age, cognitive and neurological normality at enrollment, agreement to brain donation to the UK-ADC at death, a designated informant for structured interviews, and willingness to undergo annual examinations. Participants were excluded from the cohort if they had a history of substance abuse (including alcohol), major head injury, major psychiatric illness, medical illnesses that are unstable, and/or that have an effect on the CNS, chronic infectious diseases, stroke or transient ischemic attack, encephalitis, 
meningitis, epilepsy, Parkinsonism, or other neurologic disease. The annual evaluation includes a comprehensive neuropsychological battery and general physical and neurological examinations that are detailed elsewhere [30].

Participants in the present study were 24 normal seniors without cognitive impairment and 18 seniors with aMCI (Table 1). The normal and aMCI groups did not differ in age $[t(34)=0.23, p=0.91]$. The two groups also did not differ in gender distribution $\left[\chi^{2}\right.$ $(1)=0.83, p=0.76]$, or mean number of years of education $[t(24)=0.61, p=0.86]$. All individuals receiving a diagnosis of aMCI met the following criteria: 1) a CDR score of 0.5 , with a score of at least 0.5 for memory; 2) a documented memory complaint; and 3) a score below $-1.5 \mathrm{SD}$ below their prior annual assessment on the WMS total memory score and CERAD word list delayed recall. All participants in the present study were scanned within three months of their diagnosis as normal or aMCI.

\section{FMRI task procedures}

The fMRI design included an 'active' lexical decision (LD) task and a 'passive' visual fixation task [31]. For the LD task, participants were asked to decide, as quickly and accurately as possible, if letter strings were words or pronounceable nonwords (pseudowords). For the fixation task, participants were asked to focus their vision on a centrally presented cross-hair (+). Trials were two seconds in duration. The experiment consisted of 3 separate runs, each run containing 120 trials, divided equally between the three trial types (word, nonword, fixation). The order of runs was counterbalanced across participants. An event-related design was used with trial types ordered for optimal experimental efficiency using optseq2 http://surfer.nmr.mgh.harvard.edu/optseq/.

Table 1

Subject demographics and neuropsychological scores

\begin{tabular}{lccccc}
\hline & \multicolumn{2}{c}{ aMCI } & & \multicolumn{2}{c}{ Controls } \\
\cline { 2 - 3 } \cline { 6 - 7 } & Mean & S.D. & & Mean & S.D. \\
\hline Age & 77.1 & 5.8 & & 77.9 & 7.1 \\
Education & 15.83 & 2.6 & & 16.21 & 2.4 \\
Gender & $8 \mathrm{~m} / 10 \mathrm{f}$ & & $11 \mathrm{~m} / 13 \mathrm{f}$ & \\
Clinical Dementia Rating (CDR) & 0.5 & & & 0 & \\
Mini Mental State Exam (MMSE) & $27.1^{*}$ & 1.3 & & 28.4 & 1.1 \\
WMS & $24.8^{*}$ & 5.9 & & 31.6 & 6.2 \\
CERAD Word list delayed & $4.9^{*}$ & 2.5 & & 6.9 & 1.4 \\
\hline
\end{tabular}

$* p<0.05$ statistical significance between the aMCI subjects and healthy seniors.
Stimulus presentation and recording of responses was implemented with E-Prime software (Psychology Software Tools Inc., Pittsburgh PA), using an MRI compatible projection system (SilentVision SV-6011 LCD, Avotec Inc., Stuart, FL). Visual stimuli were projected onto a screen at the back of the magnet bore, viewed by subjects through a mirror mounted on the MR head coil. Stimuli were presented in black uppercase Courier font on a white background and subtended a vertical visual angle of about $1^{\circ}$ and an average horizontal visual angle of about $4.5^{\circ}$ at the center of the field of view. Responses were made via button-presses, using a fiber-optic button-box that registers latencies to the nearest ms. Reaction times to the LD task were measured from the onset of target display. Trials with responses $\geq 2.5$ standard deviations above or below a participant's mean were excluded from RT and fMRI analyses.

\section{MRI acquisition}

Data were collected on a 3T Siemens Magnetom Trio MRI scanner, using an 8-channel head array coil, at the University of Kentucky Magnetic Resonance Imaging and Spectroscopy Center. Foam padding was used to limit head motion within the coil. Four types of image sequences were collected for each participant: 1) a standard low-resolution anatomic localizer; 2) a high-resolution, T-1 weighted sequence for the subsequent localization of fMRI activity in standard stereotactic space and assessment of group-volumetric differences; 3) T2*-weighted images sensitive to the BOLD signal for estimation of fMRI activity; 4) a $\mathrm{B}_{0}$ field map sequence for subsequent geometric unwarping of $\mathrm{T} 2 *$-weighted images.

The high-resolution, 3D anatomic image was acquired using a $\mathrm{T}-1$ weighted (MP-RAGE) image $(\mathrm{TR}=2100 \mathrm{~ms}, \quad \mathrm{TE}=2.93 \mathrm{~ms}, \quad \mathrm{TI}=1100 \mathrm{~ms}, \quad$ flip angle $=12^{\circ}, \mathrm{FOV}=224 \times 256 \times 192 \mathrm{~mm}, 1 \mathrm{~mm}$ isotropic voxels, sagittal partitions). The $\mathrm{T} 2 *$-weighted functional images were acquired in the transverse plane using a gradient-echo, EPI sequence $\left(\mathrm{TE}=30 \mathrm{~ms}, \mathrm{TR}=2000 \mathrm{~ms}\right.$, flip angle $\left.=77^{\circ}\right)$. Thirtythree interleaved slices were acquired $(64 \times 64$ image matrix, $224 \times 224 \mathrm{FOV}$, with isotropic $3.5 \mathrm{~mm}$ voxels), covering the entire cerebrum and the upper cerebellum. The $\mathrm{B}_{0}$ image used to map the spatial inhomogeneity of the $\mathrm{B}_{0}$ field consisted of a doubleecho gradient-echo sequence $(\mathrm{TE} 1=5.19 \mathrm{~ms}$, TE2 $=$ $7.65 \mathrm{~ms}$ ) with slice position and spatial resolution matching those of the EPI acquisition. 


\section{MRI analyses}

All image preprocessing and analyses (both functional and structural) were carried out using the statistical parametric mapping software package (SPM5, http://www.fil.ion.ucl.ac.uk/spm).

\section{FMRI voxel-wise analyses}

After discarding the first five functional volumes (10 s) of each run, differences in timing between slices were adjusted using the sinc interpolation. Images were then registered to the first volume of the first session using a six-parameter rigid body transformation. Next, images were unwarped via $\mathrm{B}_{0}$ field maps to reduce nonlinear magnetic field distortions. Each participant's functional images were then coregistered with their MPRAGE and were normalized into $2 \mathrm{~mm}$ isotropic voxels in MNI space using transformation parameters derived from the non-linear normalization of their MPRAGE images (described below in the VBM section). Finally, the normalized images were smoothed with an $8 \mathrm{~mm}$ FWHM Gaussian kernel and high-pass filtered with a $128 \mathrm{sec}$ cutoff period.

For the first-level individual analysis, all experimental trials were used to construct a general linear model using a canonical hemodynamic response function (HRF) with temporal and dispersion derivatives. Nuisance regressors included in the model were each run's mean, linear trend, 6 outputs from volume registration to account for residual variance from subject motion. Error trials were modeled as a separate regressor of non-interest and only correct lexical decision trials were included in the fMRI analyses. Lexical decision trials were contrasted with fixation, resulting in contrast images which were then submitted to the group level analyses.

During second-level analyses, group-based, voxelwise t-tests were performed on the mean of the two time points surrounding the peak activation in each condition (seconds 4-8 from the 14 second estimated hemodynamic response). A mixed-effect model was employed that treated condition as a fixed effect and participants as a random effect. Age, gender, and education were included as nuisance covariates in the model. Each group's 'passive' visual fixation trial types were contrasted with the 'active' LD trial types (words + nonwords). In addition, a direct betweengroup comparison was conducted to identify regions of altered task deactivation in the aMCI group. A statistical threshold of $p<0.05$ corrected for multiple comparisons at the voxel-level using the false discovery rate was employed for all comparisons. Activation maps were then projected onto semi-inflated surfaces using Caret software [32].

\section{Correlations between $R T$ and task deactivations}

Correlations were run to explore the possibility of relationships between lexical decision RT and task deactivation magnitudes in regions showing reduced deactivation in the aMCI group. ROI masks consisted of a three-dimensional area including all contiguous voxels activated $(p<0.001)$ within $10-\mathrm{mm}$ of the peak MNI coordinates of each of the three regions showing reduced deactivation in the aMCI group: left precuneus, right precuneus and left parietal cortex. ROI masks were then applied to each subject's smoothed, MNI-normalized, regressor dataset to extract the magnitude of the hemodynamic response in the baseline fixation condition compared to the LD task.

\section{Voxel-based morphometry (VBM)}

VBM preprocessing steps included segmentation, bias correction and spatial normalization, incorporated into a single generative model and thus obviating the need for creation of customized priors [33]. MRIs were segmented into grey matter (GM), white matter (WM) and cerebrospinal (CSF) images using SPM5 prior probability templates. The intensity non-uniformity bias correction was applied to aid segmentation by correcting for scanner-induced smooth, spatially varying intensity differences. GM images were normalized to their own custom templates in the standard space of the MNI T1-weighted template using a set of non-linear basis functions. A modulation step was also incorporated into the preprocessing model in order to explore regional differences in absolute volume. Intracranial volume (ICV) was estimated as the sum of GM, WM and CSF volume for each participant (for use as a nuisance covariate in subsequent statistical analysis). Finally, all normalized, modulated, GM images were smoothed an $8 \mathrm{~mm}$ FWHM isotropic Gaussian kernel.

Statistical parametric maps of differences in GM volume were determined using an analysis of covariance (ANCOVA) model with aMCI versus normal as the between factor, and age, gender, education and intracranial volume (ICV) as nuisance covariates. Second-level, group linear contrasts were then conducted on parameter estimates from the model. The voxel values for the contrasts constituted a statistical parametric map (SPM) of the $t$ statistic. Differences between groups were assessed using a statistical threshold of $p<0.05$ corrected for multiple comparisons at the voxel-level using the false discovery rate. 
Results were viewed on the canonical MNI T1 template. In addition, in order to view the overlap of volumetric and fMRI group-differences same semiinflated surfaces, the SPM T-map of regions showing decreased volume in the aMCI group was projected onto the same surfaces used to view the fMRI data, using Caret software [32].

\section{Correlations between task deactivation reductions and atrophy in aMCI}

Local atrophy-fMRI analyses explored potential relationships in the three regions in which the aMCI group showed both atrophy and DMN reductions in the voxel-wise analyses (left precuneus, right precuneus and left lateral parietal cortex). Global atrophy-fMRI analyses explored potential relationships between fMRI deactivation magnitudes in the same three regions and whole-brain volume (WBV).

Local ROI masks consisted of a three-dimensional sphere within 10-mm of the MNI center of mass coordinates of the clusters showing overlapping task deactivation reductions and atrophy in the aMCI group (Table 4). ROI masks were applied to each subject's smoothed, MNI-normalized, regressor dataset to extract fMRI task deactivation magnitudes (fixation $<$ LD). To extract VBM-based volumes, ROI masks were first applied to each subject's smoothed, MNI-normalized, modulated GM images using the spm_mask function. ROI-masked volumes (the sum of the voxels' intensities within that ROI multiplied by voxel volume) were then computed for each subject using the get_integrals script. All ROI volumes (local and WBV) were 'normalized' (divided by intracranial volume), providing an estimate of atrophy in that ROI.

A series of multiple regression analyses were conducted to explore potential relationships between normalized GM volumes and task deactivation magnitudes in the aMCI group. In each multiple regression model, fMRI task deactivation magnitude in one ROI (e.g., right precuneus) was entered as the outcome variable with normalized GM volume in the same ROI

Table 2

Peak locations and sizes of reduced task deactivation in the aMCI group compared to the normal group

\begin{tabular}{lrrrcc}
\hline Region & $\sim \mathrm{BA}$ & $X(\mathrm{~mm})$ & $Y(\mathrm{~mm})$ & $Z(\mathrm{~mm})$ & $\begin{array}{c}\text { Number of } \\
\text { voxels }\end{array}$ \\
\hline Normal>aMCI & & & & & \\
Right precuneus & 31 & 8 & -56 & 24 & 204 \\
Left precuneus & 31 & -11 & -56 & 28 & 226 \\
Left parietal & 39 & -46 & -52 & 29 & 84 \\
\hline
\end{tabular}

Note: aMCI, amnestic mild cognitive impairment; $\sim$ BA, approximate Brodmann's area.
Table 3

Peak locations and sizes of volumetric reductions in the aMCI group compared to the normal group

\begin{tabular}{lrrrrc}
\hline Region & $\sim$ BA & $X(\mathrm{~mm})$ & $Y(\mathrm{~mm})$ & $Z(\mathrm{~mm})$ & $\begin{array}{c}\text { Number of } \\
\text { voxels }\end{array}$ \\
\hline Normal > aMCI & & & & & \\
Right precuneus & 31 & 20 & -64 & 20 & 22 \\
Right precuneus* & 23 & 4 & -56 & 16 & 107 \\
Left parietal & 40 & -30 & -44 & 34 & 20 \\
Left hippocampal & & -24 & -10 & -14 & 74 \\
Left parahippo. g. & 20 & -29 & -26 & -18 & 25 \\
Right hippocampal & & 26 & -12 & -12 & 66 \\
\hline
\end{tabular}

Note: majority of cluster $*$ is located within left precuneus. aMCI, amnestic mild cognitive impairment; $\sim$ BA, approximate Brodmann's area; parahippo, parahippocampal; g., gyrus.

Table 4

Center of mass and size of clusters showing overlapping fMRI deactivation and volumetric reductions in the aMCI group

\begin{tabular}{lrrrcc}
\hline Region & $\sim \mathrm{BA}$ & $X(\mathrm{~mm})$ & $Y(\mathrm{~mm})$ & $Z(\mathrm{~mm})$ & $\begin{array}{c}\text { Number of } \\
\text { voxels }\end{array}$ \\
\hline Normal > aMCI & & & & & \\
Right precuneus & 31 & 21 & -66 & 18 & 53 \\
Left precuneus & 23 & -12 & -58 & 18 & 76 \\
Left parietal & 40 & -30 & -44 & 34 & 20 \\
\hline
\end{tabular}

Note: aMCI, amnestic mild cognitive impairment; $\sim$ BA, approximate Brodmann's area; parahippo, parahippocampal; g., gyrus.

(e.g., right precuneus), and normalized WBV as predictor variables.

\section{Receiver operating characteristic analyses}

Receiver operating characteristic (ROC) curves were generated from logistic regression models by calculating the area under the curve (AUC). ROC curves are plots of the true positive rate (sensitivity) against the false positive rate (1-specificity) for the different possible cutoff values of a classificatory variable. A total of four ROC curves were computed. Model 1 explored the classificatory accuracy of deactivation magnitudes in the three fMRI ROIs showing overlapping fMRI reductions and atrophy in the aMCI group (Table 4). Model 2 explored the classificatory accuracy of normalized volumes in the same three parietal ROIs. Model 3 explored the classificatory accuracy of the combination of models 1 and 2. Model 4 explored the classificatory accuracy of normalized volumes of bilateral hippocampal complex.

ROI masks for parietal regions were the same as those described above, in the correlation analysis section. Hippocampal volumes were obtained via ROI masks consisting of a three-dimensional sphere within 10 -mm of the MNI center of mass coordinates of the aMCI reductions observed in the whole-brain analyses (Table 3). The significance of the difference between 
the AUC for different ROC curve models was tested via the method of Hanley and McNeil [34] using MedCalc software. This method considers the following variables associated with each ROC model: the number of actual negative cases, the number of actual positive cases, the AUC, and the standard error of the AUC.

\section{RESULTS}

\section{Behavioral}

Both groups performed the LD task with very high accuracy ( $\mathrm{M}$ of the normal group $=96 \%, \mathrm{SD}=0.02$ ); $\mathrm{M}$ of the MCI group $=89 \%, \mathrm{SD}=0.15)$, although the normal group was more accurate than the MCI group $[t(40)=2.9, p<0.01]$. There was a trend for longer reaction time in the $\mathrm{MCI}$ group $(M=801 \mathrm{~ms}, \mathrm{SD}=106)$ compared to the normal group $(M=747 \mathrm{~ms}, \mathrm{SD}=73)$ $[t(40)=1.9, p=0.06]$.

\section{Voxel-wise fMRI}

Figure 1 presents statistical deactivation and activation maps for normal (panel A) and aMCI (panel B) groups. In both groups, positive activations (LD > fixation) were observed in a network of regions, prominently involving occipitotemporal cortex and portions of prefrontal cortex. In contrast, notable
A
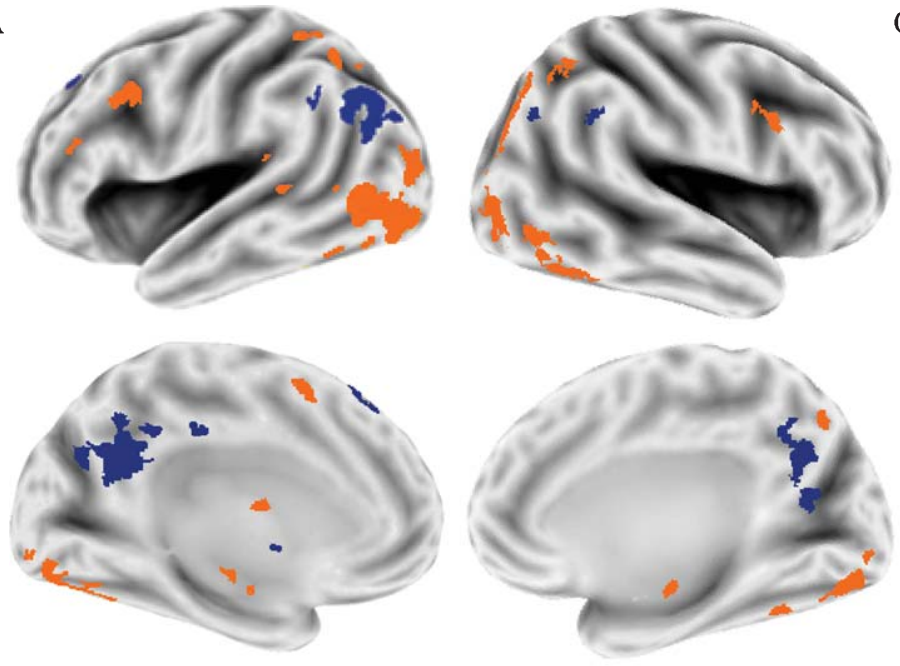

aMCI Group

B
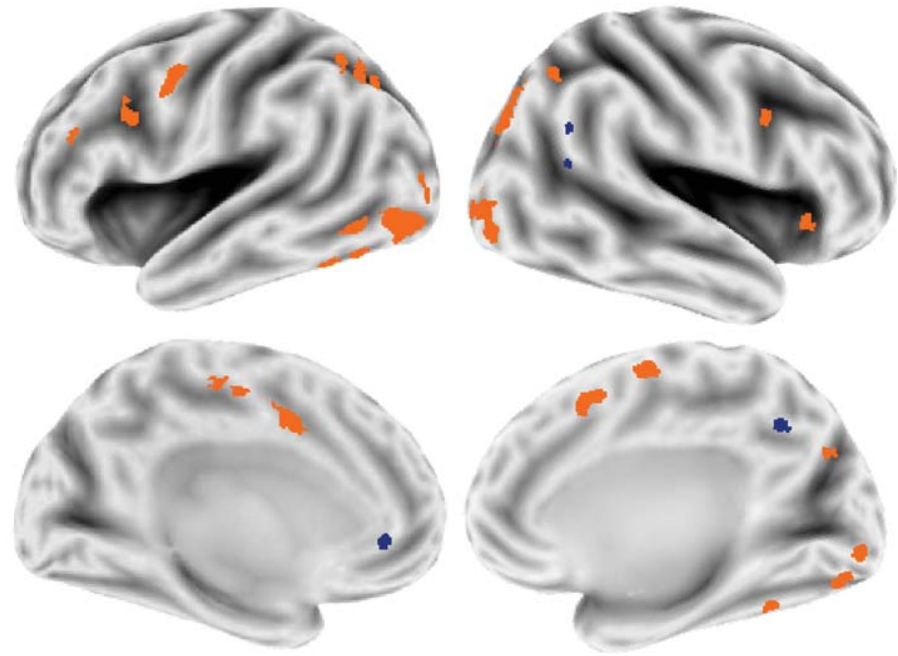

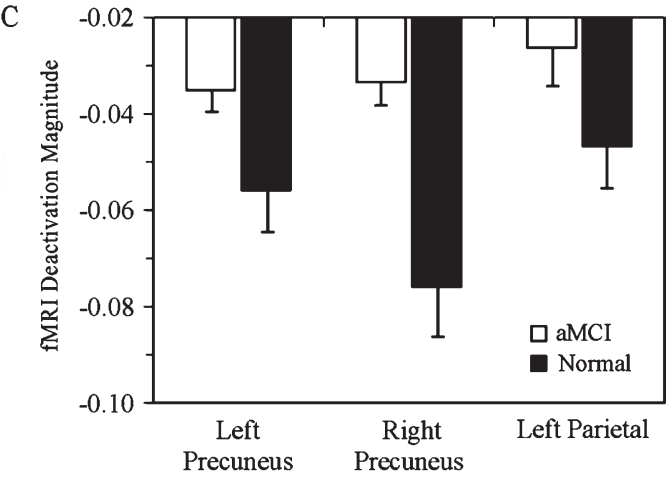

Fig. 1. Task deactivation maps. Voxel-wise maps are presented for the normal group A) and aMCI group. B) Task deactivations are presented in blue. Task activations associated with the lexical decision task are presented in orange. C) The magnitudes of deactivation (percentage signal change baseline $<$ lexical decision task) are presented for each group in the left precuneus, right precuneus and left lateral parietal cortex. 
group differences in task deactivation (deactivation; $\mathrm{LD}<$ fixation) are evident. Whereas the normal group deactivated large portions of the left lateral parietal lobe, and in bilateral regions of the precuneus, the aMCI group did not deactivate these regions.

\section{FMRI-behavioral}

There was no correlation between RT and fMRI magnitude in the left precuneus in the normal group $[r(24)=0.13, p=0.56]$ or the aMCI group $[r(18)=$ $0.09, p=0.74]$. There was no correlation between RT and fMRI magnitude in the right precuneus in the normal group $[r(24)=0.21, p=0.31]$ or the aMCI group $[r(18)=0.15, p=0.55]$. Finally, there was no correlation between RT and fMRI magnitude in the left parietal region in the normal group $[r(24)=0.26$, $p=0.23]$ or the aMCI group $[r(18)=0.19, p=0.46]$.

\section{Voxel-based morphometry}

The aMCI group showed GM volumetric reductions in bilateral hippocampi, left parahippocampal gyrus, bilateral portions of the precuneus/posterior cingulate and left lateral parietal cortex (Fig. 2). The largest cluster of reduced GM volume in the aMCI group was primarily located in the left precuneus, although its peak effect was within the right precuneus (4-56 16; 107 voxels).

\section{Overlap of task deactivation reductions and atrophy}

A direct between-group comparison confirmed reduced task deactivation in the aMCI group compared to the normal group in bilateral portions of the precuneus and left lateral parietal cortex. Fig. 3 presents the statistical maps of aMCI task deactivation reductions compared to the normal group (represented in blue) and volumetric reductions (represented in red) projected onto the same semi-inflated surfaces. The peak aMCI reductions in deactivation and volume are listed in Tables 2 and 3, respectively. It is evident that task deactivation reductions were more widespread than volumetric reductions in parietal regions, despite the fact that the average effective smoothness of the fMRI and VBM group difference
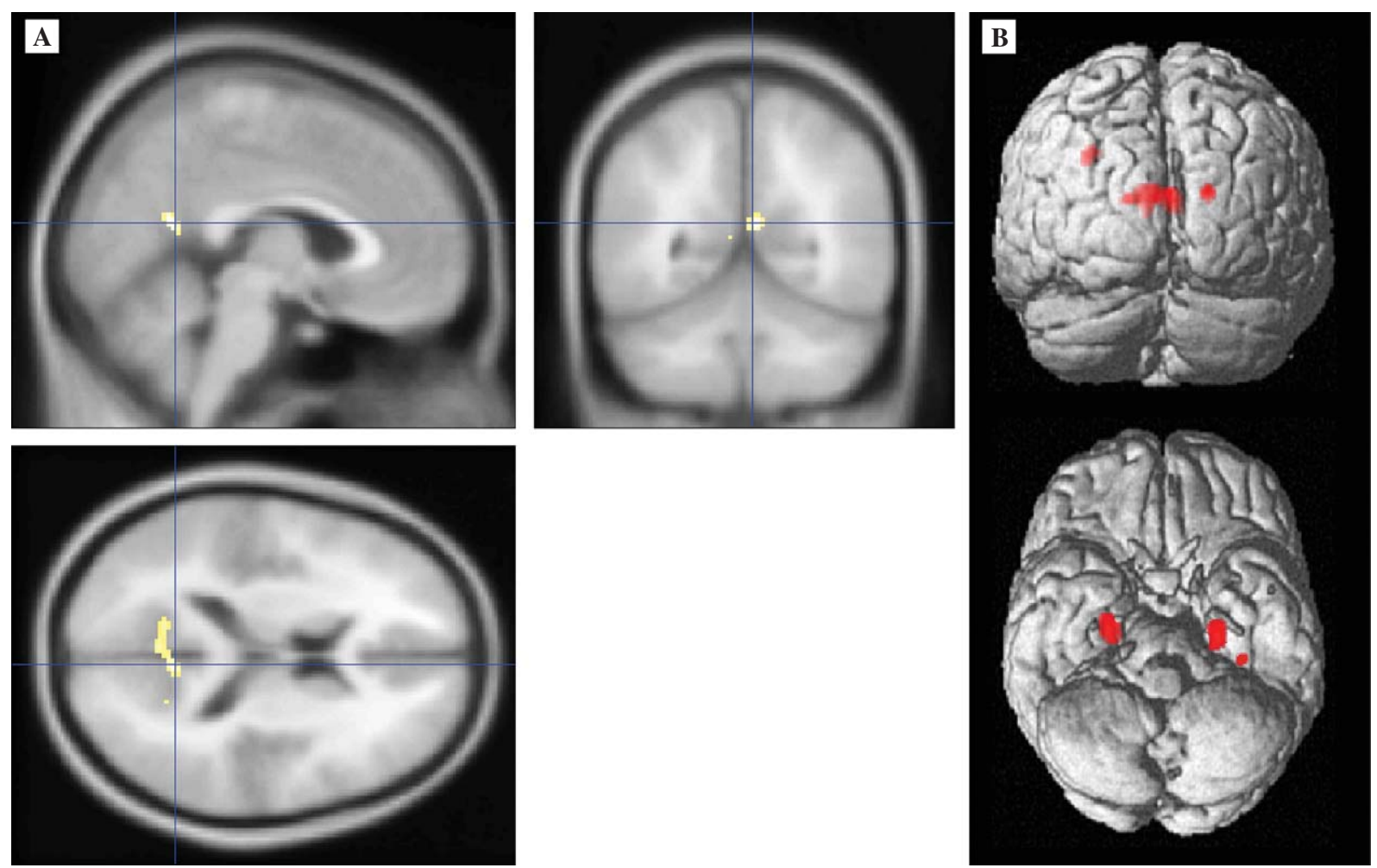

Fig. 2. Voxel-based morphometry statistical maps. Regions of significantly smaller volume in the aMCI group compared to the normal group are displayed after controlling for global intracranial differences. A) Volumetric differences highlighting a cluster within the right precuneus/posterior cingulate gyrus (crosshair) are displayed on the canonical MNI T1 template. B) The full set of volumetric differences are displayed on SPM's single subject, 3D rendered surface. 

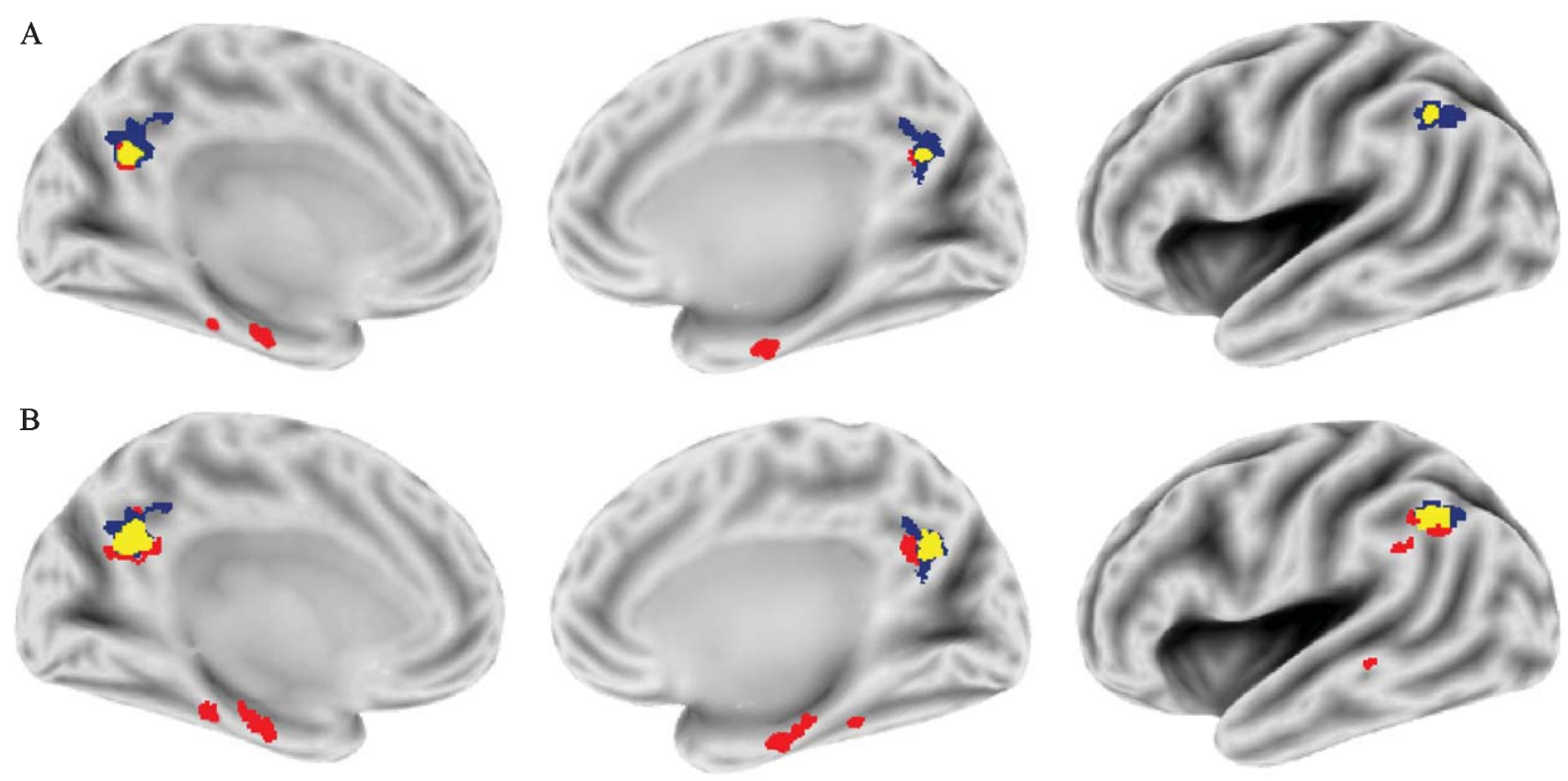

Left Medial

Right Medial

Left Lateral

DEACTIVATION $\downarrow$ ATROPHY $\uparrow$ DEACTIVATION $\downarrow$ ATROPHY $\uparrow$

Fig. 3. The overlap of task deactivation reductions and atrophy in aMCI. Direct between-group comparisons of task deactivation and volume are displayed on common semi-inflated surfaces. Regions in the aMCI group that uniquely show task deactivation reductions (blue), or atrophy (red) are displayed, as well as regions showing overlapping task deactivation reductions and atrophy (yellow). A) The use of the same multiple comparisons correction applied to fMRI and VBM data results in more widespread task deactivation reductions compared to atrophy in parietal regions. B) The use of a less stringent VBM threshold $(p<0.001$, uncorrected) results in increased overlap of volumetric declines with task deactivation reductions in parietal regions.

maps were almost identical (fMRI = 9.1 mm FWHM; $\mathrm{VBM}=8.8 \mathrm{~mm}$ FWHM).

In order to assess whether the more widespread task deactivation reductions compared to atrophy were related to greater sensitivity of the fMRI technique, a less stringent VBM threshold was employed ( $p<0.001$, uncorrected). As can be seen in Fig. 3 (B), the use of a less stringent VBM threshold tended to increase the cluster sizes of aMCI volume reductions observed at the more stringent threshold.

\section{Multiple regression analyses}

The multiple regression analyses indicated that none of the volumetric ROIs were associated with fMRI deactivation magnitudes in the aMCI group. Correlation scattergrams of fMRI activation versus GM volume are presented in Fig. 4. The fMRI deactivation magnitude in the left precuneus was uncorrelated with left precuneus GM volume $(T=-0.59, p=0.54)$ or whole brain volume $(T=-0.76, p=0.42)$. The fMRI deactivation magnitude in the right precuneus was uncorrelated with right precuneus GM volume $(T=-0.44, p=0.69)$ or whole brain volume $(T=-1.19, p=0.27)$. The fMRI deactivation magnitude in the left parietal cortex was uncorrelated with left parietal GM volume $(T=-0.91, p=0.26)$ or whole brain volume $(T=-0.87, p=0.39)$.

\section{Receiver operating characteristic analyses}

The AUC for each of the four models is presented in Fig. 5. The AUC was 0.77 for the model including fMRI deactivation magnitudes in the three parietal ROIs (left precuneus, right precuneus and left lateral parietal cortex). The AUC was 0.74 for the atrophy model that considered normalized GM volumes in these same three parietal regions. When parietal fMRI and parietal structural ROIs were combined into a single model, discriminatory accuracy increased to 0.92 . The AUC was 0.85 for the atrophy model that considered normalized GM volumes in bilateral hippocampal complex ROIs. The AUC was significantly higher for the combined parietal fMRI-atrophy model than it was for either the parietal fMRI model $(Z=1.70, p<0.05)$ or the parietal atrophy model $(Z=1.94, p<0.05)$. The 

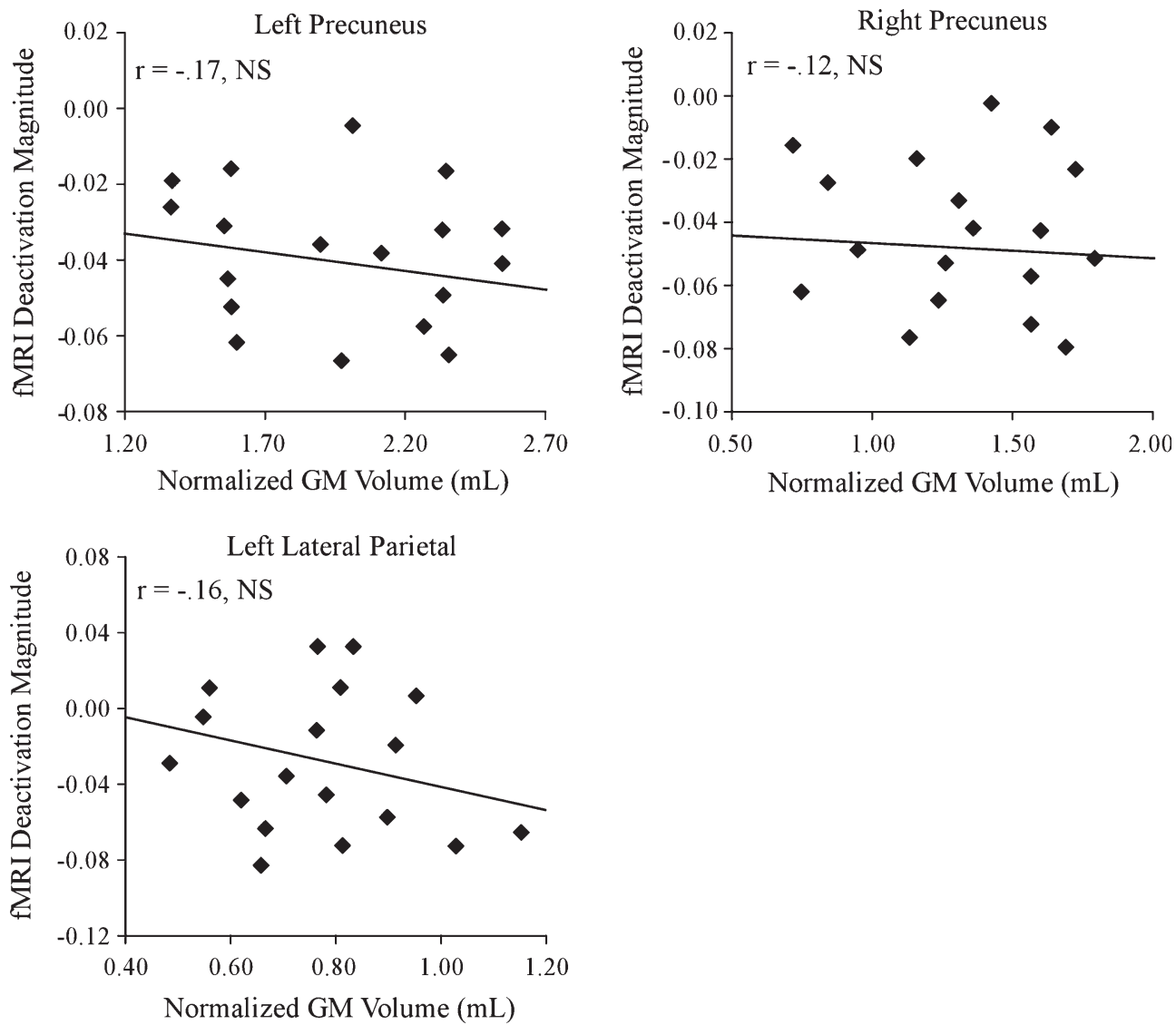

Fig. 4. The relationship between task deactivation and atrophy in parietal regions in aMCI. Regression plots display fMRI deactivation magnitude (percentage signal change fixation < lexical decision task) against normalized GM volume in three regions. Each diamond represents one of the 18 MCI participants. Note the lack of significant relationships between task deactivation and atrophy across parietal DMN regions.

AUC for the combined parietal fMRI-atrophy model was not significantly higher than the hippocampal atrophy model $(Z=1.89, p=0.19)$. However, adding hippocampal complex volumes to the combined fMRIatrophy parietal model did not improve the AUC, which decreased slightly to 0.91 .

\section{DISCUSSION}

As task deactivations reductions within the defaultmode network (DMN) become increasingly used in the study of early AD states, it is important to understand their relationship to atrophy. Our results identify multiple regions within parietal cortex as convergence areas of task deactivation reductions and atrophy in aMCI. However, results suggest that task deactivation reductions and local/global atrophy are minimally correlated in aMCI. In addition, results suggest that classificatory accuracy of aMCI is increased through the joint consideration of task deactivation reductions and atrophy within parietal nodes of the DMN. These findings converge on the conclusion that task deactivations and atrophy within parietal DMN regions provide complementary rather than redundant information in aMCI. The details of these findings are discussed below.

The present result of reduced fMRI deactivation in medial and lateral parietal regions using a LD task is consistent with results from previous studies using other tasks in aMCI [11-13]. As can be seen in Fig. 1, the failure to deactivate parietal lobe regions of the DMN in the aMCI group was not the result of positive activation in these regions during the LD task. Instead, the aMCI group showed reduced deactivation rather than positive LD activation in these regions (Fig. 1, panel C). We considered the possibility that aMCI performance reductions on the LD task may have contributed to their reduced fMRI deactivations. The small but significant reductions in aMCI accuracy would not affect their deactivations because only correct trials were included in the present event-related fMRI analyses. In contrast, the trend toward longer aMCI RTs could potentially contribute to attenuation of their 
A
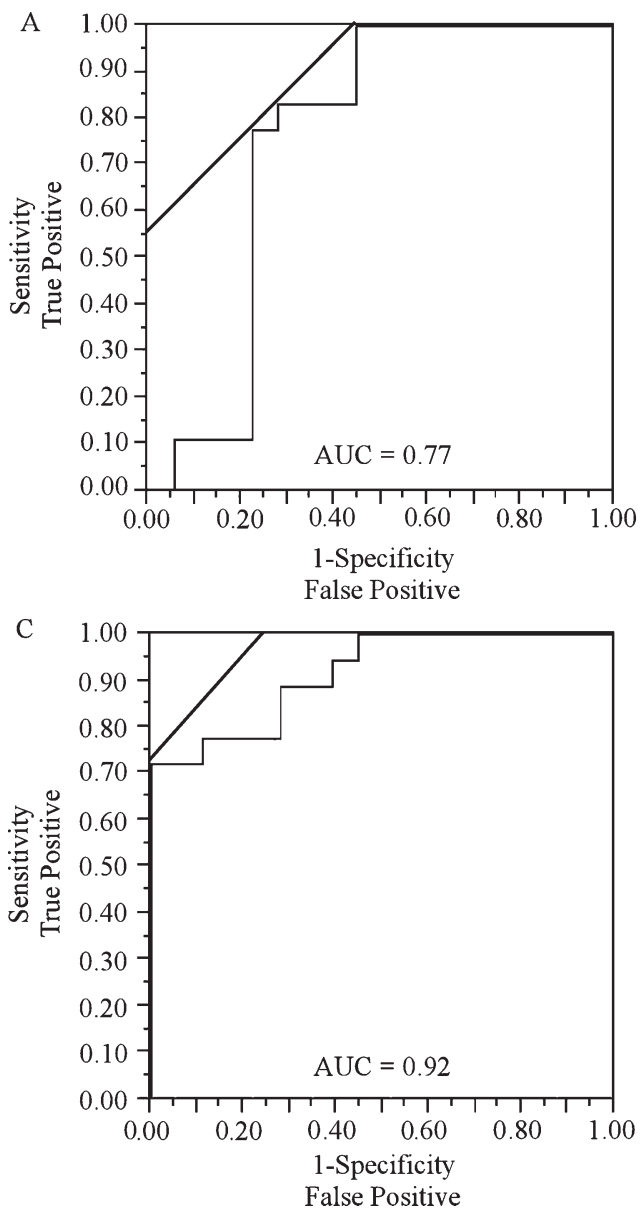
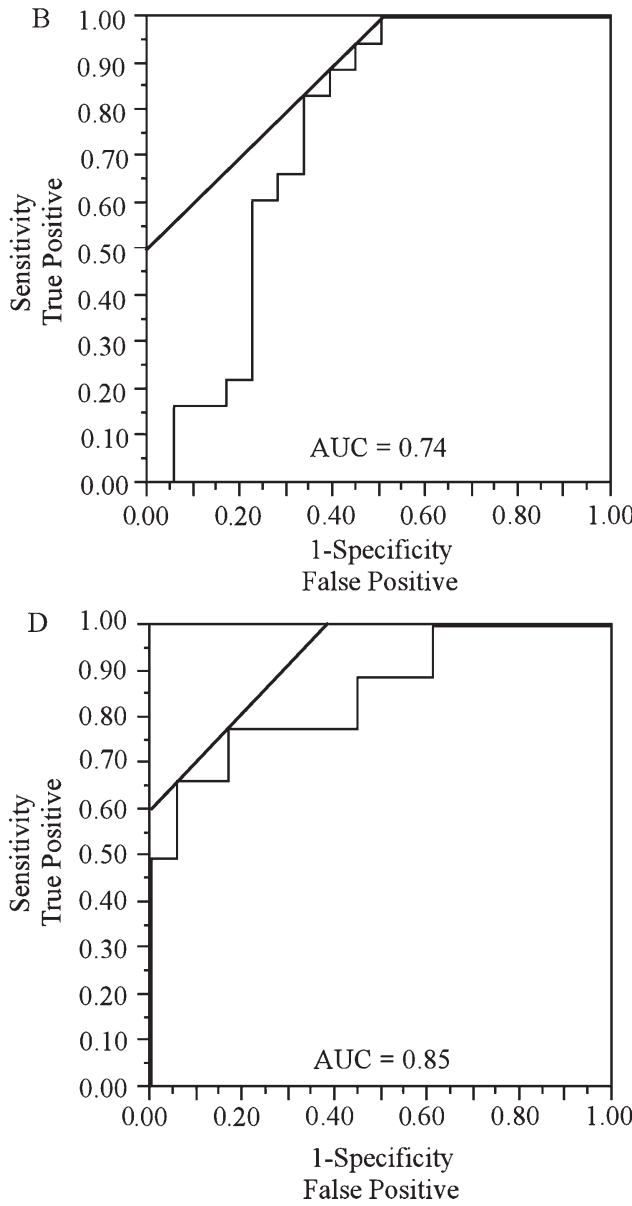

Fig. 5. Receiver operator characteristic (ROC) curves. ROC curves plot the sensitivity on the y-axis against 1-specificity on the $\mathrm{x}$-axis. The closer the curve follows the left-hand border and then the top border of the ROC space, the more accurate the test as indicated by the area under the curve (AUC). Lines at 45 degree angles tangent to the ROC curve mark the best cutoff point under the assumption that false negatives and false positives have similar costs. The ROC curves correspond to models of (A) fMRI task deactivations in three parietal ROIs (left precuneus, right precuneus and left lateral parietal cortex), (B) normalized volumes in the same three parietal regions, (C) a combined fMRI and normalized volume model in parietal regions, and (D) normalized volume in bilateral hippocampal complex. The highest classificatory accuracy was obtained for the combined fMRI and normalized volume model in parietal regions $(\mathrm{AUC}=0.92)$.

fMRI deactivations. However, results indicated no correlations between LD-RT and activation in parietal regions in the aMCI or normal group. The lack of correlation suggests that any potential relationship between task deactivations and behavioral performance on the present LD task appears to be weak.

The VBM analysis demonstrated aMCI atrophy in medial/lateral portions of parietal cortex in addition to medial temporal lobe regions, consistent with previous reports of relatively circumscribed GM loss in aMCI [25-27]. Moreover, projection of voxel-wise fMRI and volumetric results to a common surface showed that each of the parietal DMN regions showing reduced task deactivation also showed significant atrophy in the aMCI group. The regional pattern of topograph- ical overlap observed suggests that some AD-based pathophysiological processes that affect function and structure have a predilection for parietal nodes of the DMN early in the disease process. This is consistent with results showing prominent amyloid deposition in DMN regions [35]. In addition, results are consistent with studies showing that amyloid deposition in DMN regions disrupts task deactivation [36], functional connectivity [37], and neuropsychological performance [38].

Although task deactivation reductions and atrophy showed topographical overlap in the present study, task deactivation reductions tended to be more widespread within parietal regions than atrophy in these regions (Fig. 3, panel A). The more widespread task deacti- 
vation reductions were observed despite very similar processing pipelines of fMRI and volumetric data, including application of the same non-linear spatial normalization algorithms, virtually identical average effective smoothness of the fMRI and VBM group difference maps, use of the same nuisance covariates (age, gender, education) and application of the same (corrected) statistical threshold. However, when a less stringent threshold ( $p<0.001$ uncorrected) was employed for the VBM comparison, an increase in the overlap of volumetric declines with task deactivation reductions was observed in parietal and MTL regions (Fig. 3, panel B). We interpret this finding as evidence that task deactivation reductions appear to be a more sensitive indicator of aMCI neural declines in parietal regions than volumetric reductions. However, it should be pointed out that it is not possible to determine whether this increased sensitivity relates to technical advantages of fMRI or actual underlying disease processes without autopsy or brain biopsy data.

Results from multiple regression analyses indicated a minimal association between task deactivation reductions and atrophy in parietal regions. The relationship between fMRI signal and atrophy is likely to be complex and non-linear, and there are several possible reasons for the lack of correlation we observed. One possibility is that fMRI reductions and atrophy may be differentially sensitive to pathological features of aMCI. Another possibility takes into account the potentially opposing influences of partial volume effects and neural compensation on the fMRI signal. For example, atrophy would be expected to increase partial volume effects which, in turn, would be expected to decrease fMRI signal due to the increased amount of cerebrospinal fluid within voxels. On the other hand, positive correlations have been reported between fMRI signal and atrophy in both neighboring regions [39], and remote regions [40], which may reflect compensation. In the present study, these variables may have offset each other. In more advanced AD states, negative correlations between fMRI signal and local atrophy have been reported [41, 42], possibly because compensation may become less viable as $\mathrm{AD}$ progresses.

Results from ROC classification analyses provide convergent evidence for several findings from the present study. The model that explored magnitudes of fMRI deactivations in parietal ROIs differentiated the aMCI and normal groups with $77 \%$ accuracy, compared to $74 \%$ accuracy for the model which explored structural volumes in the same regions. This ROC finding (reflecting magnitude) is consistent with our observed whole-brain results (reflecting spatial extent), which suggests that fMRI appears to provide a more sensitive measure of aMCI neural declines in parietal regions than VBM. However, when fMRI and volumetric parietal ROIs were combined into a single model, discriminatory accuracy increased to $92 \%$. This finding is consistent with correlation results suggesting that fMRI reductions and atrophy in parietal nodes of the DMN provide complementary rather than redundant information in aMCI.

We note several caveats that highlight areas for future investigation. First, future multimodal imaging studies using a longitudinal design will be required to unravel the relative timing of task deactivation reductions and medial temporal lobe atrophy. This issue is of particular importance in light of findings that task deactivation reductions have been reported in cognitive normal seniors at high genetic risk for $\mathrm{AD}[9,43]$, and reduced medial temporal lobe volumes have been reported in normal seniors several years prior to a diagnosis of aMCI [44]. Second, although most individuals given a clinical diagnosis of aMCI at our center show AD pathology [15], it is not yet possible to know what percentage of the present aMCI or normal samples harbor significant AD pathology. Some misclassification of participants (according to the neuropathology gold standard) is thus possible. This issue should be addressable in the future because participants in the longitudinal normal cohort study at our ADRC have agreed to brain donation at death.

In conclusion, our results represent progress toward understanding the relationship between task deactivation reductions and atrophy within the DMN. Our results identify multiple regions within parietal cortex as convergence areas of task deactivation reductions and atrophy in aMCI, suggesting that some pathophysiological processes that affect function and structure have a predilection for parietal DMN regions. However, results from multiple analyses converge on the conclusion that task deactivation reductions and atrophy provide complementary rather than redundant information in aMCI. Future research will be required to assess the utility of task deactivation reductions and atrophy within the DMN in the detection of early stages of AD.

\section{ACKNOWLEDGMENTS}

This work was supported by the National Institutes of Health (NIA Grant R01 AG033036) and the National Science Foundation (BCS Grant 0814302). The authors thank Jeff Covell for help with data col- 
lection, Dr. Chobok Kim for technical support, and Dr. Richard Kryscio for statistical support. In addition, we thank three anonymous reviewers for helpful comments on an earlier version of this manuscript.

Authors' disclosures available online http://www.jalz.com/disclosures/view.php?id=923.

\section{REFERENCES}

[1] Binder JR, Frost JA, Hammeke TA, Bellgowan PS, Rao SM, Cox RW (1999) Conceptual processing during the conscious resting state. A functional MRI study. J Cogn Neurosci 11, $80-95$.

[2] Mazoyer B, Zago L, Mellet E, Bricogne S, Etard O, Houde O, Crivello F, Joliot M, Petit L, Tzourio-Mazoyer N (2001) Cortical networks for working memory and executive functions sustain the conscious resting state in man. Brain Res Bull 54, 287-298.

[3] Shulman GL, Fiez JA, Corbetta M, Buckner RL, Miezin FM, Raichle ME, Petersen SE (1997) Common blood flow changes across visual tasks. 2. Decreases in cerebral cortex. J Cogn Neurosci 9, 648-663.

[4] Buckner RL, Andrews-Hanna JR, Schacter DL (2008) The brain's default network - Anatomy, function, and relevance to disease. Year Cogn Neurosci 1124, 1-38.

[5] Raichle ME, MacLeod AM, Snyder AZ, Powers WJ, Gusnard DA, Shulman GL (2001) A default mode of brain function. Proc Natl Acad Sci U S A 98, 676-682.

[6] Damoiseaux JS, Rombouts SA, Barkhof F, Scheltens P, Stam CJ, Smith SM, Beckmann CF (2006) Consistent resting-state networks across healthy subjects. Proc Natl Acad Sci U S A 103, 13848-13853.

[7] Greicius MD, Krasnow B, Reiss AL, Menon V (2003) Functional connectivity in the resting brain: a network analysis of the default mode hypothesis. Proc Natl Acad Sci U S A 100, 253-258.

[8] Fox MD, Snyder AZ, Vincent JL, Corbetta M, Van Essen DC, Raichle ME (2005) The human brain is intrinsically organized into dynamic, anticorrelated functional networks. Proc Natl Acad Sci U S A 102, 9673-9678.

[9] Smith CD, Andersen AH, Kryscio RJ, Schmitt FA, Kindy MS, Blonder LX, Avison MJ (2002) Women at risk for AD show increased parietal activation during a fluency task. Neurology 58, 1197-1202.

[10] Lustig C, Snyder AZ, Bhakta M, O’Brien KC, McAvoy M, Raichle ME, Morris JC, Buckner RL (2003) Functional deactivations: Change with age and dementia of the Alzheimer type. Proc Natl Acad Sci U S A 100, 14504-14509.

[11] Celone KA, Calhoun VD, Dickerson BC, Atri A, Chua EF, Miller SL, DePeau K, Rentz DM, Selkoe DJ, Blacker D, Albert MS, Sperling RA (2006) Alterations in memory networks in mild cognitive impairment and Alzheimer's disease: An independent component analysis. J Neurosci 26, 1022210231.

[12] Rombouts SA, Barkhof F, Goekoop R, Stam CJ, Scheltens $P$ (2005) Altered resting state networks in mild cognitive impairment and mild Alzheimer's disease: An fMRI study. Hum Brain Mapp 26, 231-239.

[13] Petrella JR, Wang L, Krishnan S, Slavin MJ, Prince SE, Tran TT, Doraiswamy PM (2007) Cortical deactivation in mild cognitive impairment: High-field-strength functional MR imaging. Radiology 245, 224-235.
[14] Jicha GA, Parisi JE, Dickson DW, Johnson K, Cha R, Ivnik RJ, Tangalos EG, Boeve BF, Knopman DS, Braak H, Petersen RC (2006) Neuropathologic outcome of mild cognitive impairment following progression to clinical dementia. Arch Neurol 63, 674-681.

[15] Markesbery WR, Schmitt FA, Kryscio RJ, Davis DG, Smith CD, Wekstein DR (2006) Neuropathologic substrate of mild cognitive impairment. Arch Neurol 63, 38-46.

[16] Morris JC, Storandt M, Miller JP, McKeel DW, Price JL, Rubin EH, Berg L (2001) Mild cognitive impairment represents early-stage Alzheimer disease. Arch Neurol 58, 397-405.

[17] Petersen RC, Doody R, Kurz A, Mohs RC, Morris JC, Rabins PV, Ritchie K, Rossor M, Thal L, Winblad B (2001) Current concepts in mild cognitive impairment. Arch Neurol 58, 19851992.

[18] Friedland RP, Budinger TF, Ganz E, Yano Y, Mathis CA, Koss B, Ober BA, Huesman RH, Derenzo SE (1983) Regional cerebral metabolic alterations in dementia of the Alzheimer type - positron emission tomography with [fluorodeoxyglucose-F18. J Comp Asst Tomogr 7, 590-598.

[19] Herholz K (1995) FDG PET and differential-diagnosis of dementia. Alz Dis Assoc Disord 9, 6-16.

[20] Reiman EM, Caselli RJ, Yun LS, Chen KW, Bandy D, Minoshima S, Thibodeau SN, Osborne D (1996) Preclinical evidence of Alzheimer's disease in persons homozygous for the epsilon 4 allele for apolipoprotein E. New Engl J Med 334, 752-758.

[21] Baron JC, Chetelat G, Desgranges B, Perchey G, Landeau B, de la Sayette V, Eustache F (2001) In vivo mapping of gray matter loss with voxel-based morphometry in mild Alzheimer's disease. Neuroimage 14, 298-309.

[22] Gold BT, Balota D, Cortese MJ, Sergent-Marshall SD, Snyder AZ, Salat DH, Fischl B, Dale AM, Morris JC, Buckner RL (2005) Differing neuropsychological and neuroanatomical correlates of abnormal reading in early-stage semantic dementia and dementia of the Alzheimer type. Neuropsychologia 43, 833-846.

[23] Scahill RI, Schott JM, Stevens JM, Rossor MN, Fox NC (2002) Mapping the evolution of regional atrophy in Alzheimer's disease: Unbiased analysis of fluid-registered serial MRI. Proc Natl Acad Sci U S A 99, 4703-4707.

[24] Thompson PM, Hayashi KM, de Zubicaray G, Janke AL, Rose SE, Semple J, Herman D, Hong MS, Dittmer SS, Doddrell DM, Toga AW (2003) Dynamics of gray matter loss in Alzheimer's disease. J Neurosci 23, 994-1005.

[25] Chetelat G, Desgranges B, de la Sayette V, Viader F, Eustache F, Baron JC (2002) Mapping gray matter loss with voxel-based morphometry in mild cognitive impairment. $\mathrm{Neu}$ roreport 13, 1939-1943.

[26] Hamalainen A, Tervo S, Grau-Olivares M, Niskanen E, Pennanen C, Huuskonen J, Kivipelto M, Haenninen T, Tapiola M, Vanhanen M, Hallikainen M, Helkala EL, Nissinen A, Vanninen R, Soininen H (2007) Voxel-based morphometry to detect brain atrophy in progressive mild cognitive impairment. Neuroimage 37, 1122-1131.

[27] Karas GB, Scheltens P, Rombouts SARB, Visser PJ, van Schijndel RA, Fox NC, Barkhof F (2004) Global and local gray matter loss in mild cognitive impairment and Alzheimer's disease. Neuroimage 23, 708-716.

[28] He Y, Wang L, Zang YF, Tian LX, Zhang XQ, Li KC, Jiang TZ (2007) Regional coherence changes in the early stages of Alzheimer's disease: A combined structural and resting-state functional MRI study. Neuroimage 35, 488-500.

[29] Bai F, Zhang ZJ, Yu H, Shi YM, Yuan YG, Zhu WL, Zhang XR, Qian Y (2008) Default-mode network activity 
distinguishes amnestic type mild cognitive impairment from healthy aging: A combined structural and resting-state functional MRI study. Neurosci Lett 438, 111-115.

[30] Schmitt FA, Wetherby MMC, Wekstein DR, Dearth CMS, Markesbery WR (2001) Brain donation in normal aging: Procedures, motivations, and donor characteristics from the biologically resilient adults in neurological studies (BRAiNS) project. Gerontologist 41, 716-722.

[31] Gold BT, Jiang Y, Jicha GA, Smith CD (2010) Functional response in ventral temporal cortex differentiates mild cognitive impairment from normal aging. Hum Brain Mapp 31, 1249-1259.

[32] Van Essen DC, Drury HA, Dickson J, Harwell J, Hanlon D, Anderson $\mathrm{CH}$ (2001) An integrated software suite for surfacebased analyses of cerebral cortex. J Am Med Inform Assoc 8, 443-459.

[33] Ashburner J, Friston KJ (2005) Unified segmentation. Neuroimage $\mathbf{2 6}, 839-851$

[34] Hanley JA, Mcneil BJ (1982) The meaning and use of the area under a receiver operating characteristic (Roc) curve. Radiology 143, 29-36.

[35] Buckner RL, Snyder AZ, Shannon BJ, LaRossa G, Sachs R, Fotenos AF, Sheline YI, Klunk WE, Mathis CA, Morris JC, Mintun MA (2005) Molecular, structural, and functional characterization of Alzheimer's disease: Evidence for a relationship between default activity, amyloid, and memory. $J$ Neurosci 25, 7709-7717.

[36] Sperling RA, LaViolette PS, O'Keefe K, O'Brien J, Rentz DM, Pihlajamaki M, Marshall G, Hyman BT, Selkoe DJ, Hedden T, Buckner RL, Becker JA, Johnson KA (2009) Amyloid deposition is associated with impaired default network function in older persons without dementia. Neuron 63, 178-188

[37] Hedden T, Van Dijk KRA, Becker JA, Mehta A, Sperling RA, Johnson KA, Buckner RL (2009) Disruption of func- tional connectivity in clinically normal older adults harboring amyloid burden. J Neurosci 29, 12686-12694.

[38] Rentz DM, Locascio JJ, Becker JA, Moran EK, Eng E, Buckner RL, Sperling RA, Johnson KA (2010) Cognition, reserve, and amyloid deposition in normal aging. Ann Neurol 67, 353364.

[39] Johnson SC, Saykin AJ, Baxter LC, Flashman LA, Santulli RB, McAllister TW, Mamourian AC (2000) The relationship between fMRI activation and cerebral atrophy: Comparison of normal aging and alzheimer disease. Neuroimage 11, 179187.

[40] Dickerson BC, Salat DH, Greve DN, Chua EF, RandGiovannetti E, Rentz DM, Bertram L, Mullin K, Tanzi RE, Blacker D, Albert MS, Sperling RA (2005) Increased hippocampal activation in mild cognitive impairment compared to normal aging and AD. Neurology 65, 404-411.

[41] Prvulovic D, Hubl D, Sack AT, Melillo L, Maurer K, Frolich L, Lanfermann H, Zanella FE, Goebel R, Linden DE, Dierks $\mathrm{T}$ (2002) Functional imaging of visuospatial processing in Alzheimer's disease. Neuroimage 17, 1403-1414.

[42] Remy F, Mirrashed F, Campbell B, Richter W (2005) Verbal episodic memory impairment in Alzheimer's disease: A combined structural and functional MRI study. Neuroimage 25, 253-266

[43] Persson J, Lind J, Larsson A, Ingvar M, Sleegers K, Van Broeckhoven C, Adolfsson R, Nilsson LG, Nyberg L (2008) Altered deactivation in individuals with genetic risk for Alzheimer's disease. Neuropsychologia 46, 1679-1687.

[44] Martin SB, Smith CD, Collins HR, Schmitt FA, Gold BT (2010) Evidence that volume of anterior medial temporal lobe is reduced in seniors destined for mild cognitive impairment. Neurobiol Aging 31, 1099-1106. 